\title{
Orientation insensitive UHF RFID Tag Antenna with polarization diversity using Characteristic Mode Analysis
}

\author{
Abubakar Sharif,Jun Ouyang \\ School of Electronic Science and Engineering \\ University of Electronic Science and Technology of China \\ Chengdu,China \\ sharifuetsc@gmail.com
}

\author{
Muhammad Ali Imran,Qammer H. Abbasi \\ Dept. of Electronics and Nanoscale Engineering \\ University of Glasgow \\ Glasgow, UK \\ qammer.abbasi@glasgow.ac.uk
}

\begin{abstract}
This paper presents a UHF RFID tag antenna design with polarization diversity using characteristic mode analysis. The proposed tag antenna consists of two meander dipole-like structure and shorting stubs. By analyzing characteristic modes, the diagonal slots are created to scale down the resonating modes. Since the modes are depicting inductive behavior in the frequency band from $902 \mathrm{MHz}$ to $928 \mathrm{MHz}$, therefore, the small capacitive slots are created to achieve mode resonance in the required RFID band. Moreover, the proposed tag design is low-cost due to the absence of vias and provide a bandwidth of more than $40 \mathrm{MHz}$ with a 3D orientation insensitive read range pattern. Furthermore, the proposed tag design is able to provide a read range of $7.5 \mathrm{~m}$ and $4 \mathrm{~m}$ on a metal plate and low permittivity dielectric materials.
\end{abstract}

\section{INTRODUCTION}

Radio frequency identification has been emerging in numerous identification and sensing applications especially in collaboration with the internet of things (IoT). The application areas of RFID include supply chain management, retail management, tracking and monitoring of patients in hospitals and etc. These vast applications require tagging of many challenging environment surfaces such as metal, wood, water, and other high permittivity materials. The most of conventional and commercial tags are consists of dipole type structures posing a linear polarization, which further leads to orientation sensitivity and quasi-omnidirectional radiation pattern. Usually, the RFID system employs a circularly polarized reader antenna to solve the orientation sensitivity problem at the expense of $3 \mathrm{~dB}$ loss due to polarization mismatch between the reader antenna and tag [1]-[2]. In [3], a dual polarized PIFA based RFID tag antenna was proposed to solve the orientation problem, however, the design has large dimensions $64 \times 64 \times 2 \mathrm{~mm} 3$. Moreover, this invention of 12 vias and lumped elements have made this design very complex, because the resonant frequency is highly depending on the location of vias. Another solution was proposed in [4], although this proposed design is small, however, it is posing a very small bandwidth especially the real impedance is very small ( 3 to $4 \mathrm{Ohms}$ ). Therefore, in this paper, a UHF RFID tag antenna with polarization sensitivity using CMA is
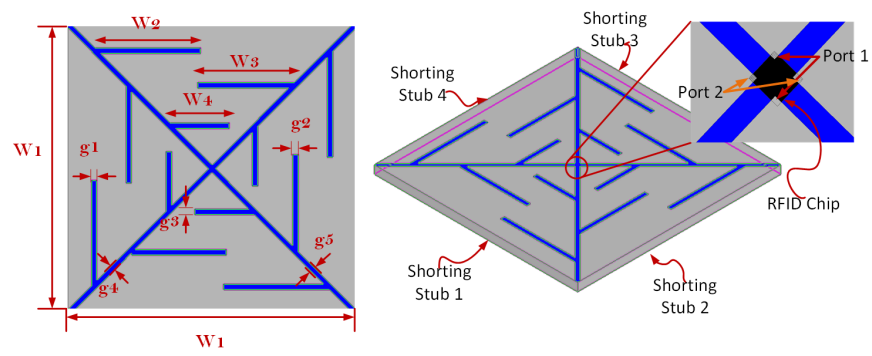

Fig. 1. Geometry and detailed dimensions of Proposed RFID $\operatorname{tag}(\mathrm{W} 1=50, \mathrm{~W} 2=18.36, \mathrm{~W} 3=16.86, \mathrm{~W} 4=10.36, \mathrm{~g} 1=\mathrm{g} 2=\mathrm{g} 3=\mathrm{g} 4=\mathrm{g} 5=1)$.

presented to address the orientation sensitivity problem. The proposed tag poses a polarization diversity to achieve true 3D read range pattern with increased read range (with linearly polarized reader antenna), even on metallic objects. The tag consists of two meander dipole-like structure and shorting stubs. By analyzing characteristic modes, the diagonal slots are created to scale down the resonating modes. Since the modes are depicting inductive behavior in the frequency band from $902 \mathrm{MHz}$ to $928 \mathrm{MHz}$, accordingly, the small capacitive slots are created to achieve mode resonance in required RFID band. The antenna parameters are further optimized using CST to achieve a conjugate match with Impinj Monza 4 D RFID chip. Furthermore, the proposed tag design is able to provide a read range of $7.5 \mathrm{~m}$ and $4 \mathrm{~m}$ on $200 \times 200 \mathrm{~mm} 2$ metal plate and low permittivity dielectric materials.

\section{Concept And Antenna Design using CMA}

Fig. 1 shows the geometry and detailed dimensions of the proposed RFID tag antenna. The tag antenna was based on inexpensive FR4 substrate and consists of diagonal and parallel slots carefully created to scale down the mode resonant frequency using CMA. Starting with CMA of $50 \times 50$ metallic plate placed on a grounded substrate and shorted from each end to the ground with shorting stubs, it is found that more than four modes are resonating between 3 to $4 \mathrm{GHz}$. The resonating frequencies of these modes are scale down by 


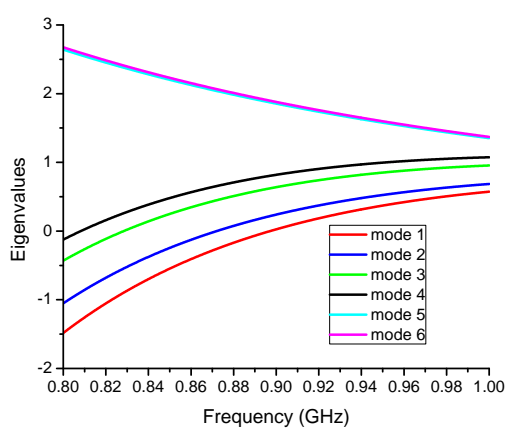

Fig. 2. Eigenvalue plot of first six modes of proposed tag antenna.

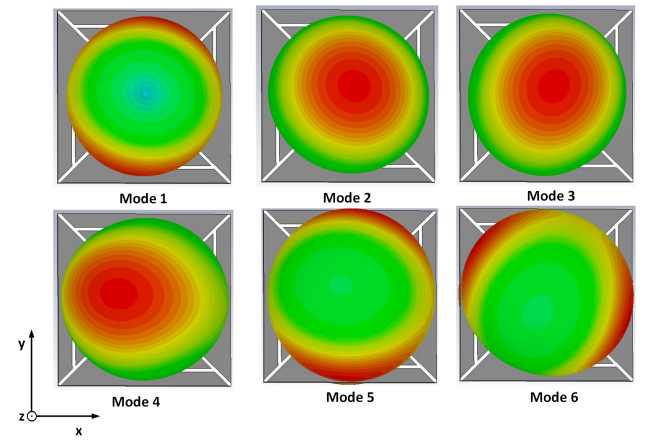

Fig. 3. Far-field radiation pattern associated with first six modes of proposed tag antenna.

creating two diagonal slots. In this case, some modes are showing inductive behavior [5] in UHF RFID frequency band $860 \mathrm{MHz}$ to $960 \mathrm{MHz}$. Consequently, the parallel slots having capacitive behavior are created to convert the inductive modes into the resonating mode in required UHF RFID band as illustrated by eigenvalue plot of first six modes of proposed tag antenna in Fig. 2. Since the mode 1,2,3 and 4 are resonating in the required band and can be exploited to achieve polarization diversity by carefully exciting two or more mode. In order to explore the mode characteristics further, the far-field radiation patterns associated with each mode are shown in Fig. 3. The mode 1 has an asymmetric current distribution which results in the cancelation of the field in the $+\mathrm{z}$ direction. However, the mode pairs $(2,4)$ and $(3,4)$ can be exploited to achieve polarization diversity. The tag parameters are optimized further using CST Microwave studio to get a conjugate match with Impinj Monza 4 D RFID chip with impedance 11-143j @ 915 MHz. Fig. 4 show the real and imaginary impedance plot of the proposed design, the real and imaginary impedance are ranging from 5 to 7 Ohms and 135 to $145 \mathrm{ohms}$ in US RFID band $(902 \mathrm{MHz}-928 \mathrm{MHz})$, respectively. Moreover, the return loss and port isolation of proposed tag design are shown in Fig. 5 . The $3 \mathrm{~dB}$ bandwidth of the proposed design is more than $40 \mathrm{MHz}$, which is acceptable for many practical applications. Additionally, the port isolation between two port of tag design is $-45 \mathrm{~dB}$, which show the significance of this design for

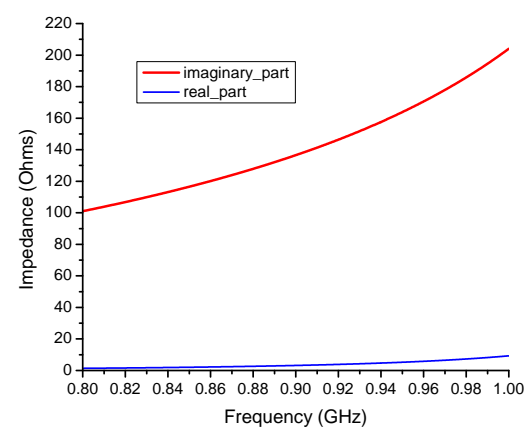

Fig. 4. Input impedance plot of two ports of proposed tag design.

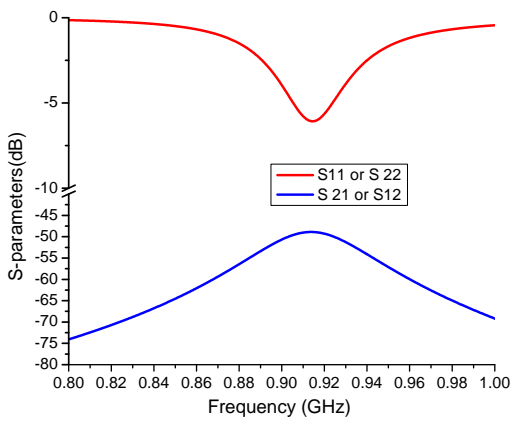

Fig. 5. S11 plot and isolation S21 between two ports.

polarization diversity and orientation sensitive applications.

\section{CONCLUSION}

An orientation insensitive UHF tag antenna with true 3-D read range pattern is presented using CMA. The proposed tag design is low-cost due to the absence of vias and provide a bandwidth of more than $40 \mathrm{MHz}$ with a 3D orientation insensitive read range pattern. Furthermore, the proposed tag design is able to provide a read range of $7.5 \mathrm{~m}$ and $4 \mathrm{~m}$ on metal plate and low permittivity dielectric materials(with linearly polarized reader antenna).

\section{REFERENCES}

[1] E. Perret, S. Tedjini, and R. S. Nair, Design of antennas for UHF RFID tags, Proc. IEEE, vol. 100, no. 7, pp. 23302340, 2012.

[2] A. Sharif, J. Ouyang, F. Yang, R. Long, and M. K. Ishfaq, Tunable Platform Tolerant Antenna Design for RFID and IoT Applications Using Characteristic Mode Analysis, Wirel. Commun. Mob. Comput., vol. 2018, 2018.

[3] L. A. Berge and M. T. Reich, A UHF RFID Antenna for a Wireless Sensor Platform with a Near-Isotropic Radiation Pattern, 2013 IEEE Int. Conf. RFID, pp. 8895, 2013.

[4] E. Yang and H. Son, Dual-polarised metal-mountable UHF RFID tag antenna for polarisation diversity, vol. 52, no. 7, pp. 45, 2016.

[5] F. L. Bong,E. H. Lim, and F. L. Lo, Compact Orientation Insensitive Dipolar Patch for, vol. 66, no. 4, pp. 17881795, 2018.

[6] M. Cabedo-Fabres, E. Antonino-Daviu, A. Valero-Nogueira, and M. F. Bataller, The theory of characteristic modes revisited: A contribution to the design of antennas for modern applications, IEEE Antennas Propag. Mag., vol. 49, no. 5, pp. 5268, 2007. 